\title{
Approximate Solutions for Some Nonlinear Evolutions Equations By Using The Reduced Differential Transform Method
}

\author{
${ }^{1}$ Birol İbiş, ${ }^{2}$ Mustafa Bayram \\ ${ }^{1}$ Department of Main Sciences, Turkish Air Force Academy, Istanbul,Turkey \\ E-mail:bibis@hho.edu.tr \\ ${ }^{2}$ Yıldız Technical University, Department of Math. Eng., Esenler,Istanbul,Turkey \\ E-mail:msbayram@yildiz.edu.tr
}

\begin{abstract}
In this paper, the reduced differential transform method (RDTM) is applied to various nonlinear evolution equations, Korteweg-de Vries Burgers' (KdVB) equation, Drinefel'd-Sokolov-Wilson equations, coupled Burgers equations and modified Boussinesq equation. Approximate solutions obtained by the RDTM are compared with the exact solutions. The present results are in good agreement with the exact solutions. Comparisons show that the RDTM is capable of solving effectively a large number of nonlinear evolution equations with high accuracy.
\end{abstract}

Keywords: Reduced Differential Transform Method (RDTM), Korteweg-de Vries Burgers' (KdVB) equation, Drinefel'd-Sokolov-Wilson equations, coupled Burgers equations and modified Boussinesq equation.

\section{Introduction}

Nonlinear evolution equations (NLEEs) are widely used to describe many important phenomena and dynamic processes in physics, mechanics, chemistry, biology, etc. The investigation of exact solutions of NLEEs plays an important role in the study of nonlinear physical phenomena. There has been a great amount of activity aiming to find methods for solutions of NLEEs. Recently many new approaches to NLEEs have been proposed, for example, the variational iteration method [1-3], the homotopy perturbation method [3-6], various tanh function 
methods [7-11], the F-expansion method [12-13], the sine-cosine method [1417], Hirota method [18,19], Jacobi elliptic function method [20-22], homogeneous balance method [23-24], the $\left(\mathrm{G}^{\prime} / \mathrm{G}\right)$-expansion method [25-27] and the expfunction method [28-31].

Keskin in [32] introduced a reduced form of differential transform method (DTM) as reduced differential transform method (RDTM) and applied to approximate some PDEs and fractional PDEs [33-34]. Abazari and Ganji [35] extended RDTM to study the partial differential equation with proportional delay in $\mathrm{t}$ and shrinking in $\mathrm{x}$, and shown that as a special advantage of RDTM rather than DTM. The reduced differential transform recursive equations produce exactly all the Poisson series coefficients of solutions, whereas the differential transform recursive equations produce exactly all the Taylor series coefficients of solutions.

In this paper, we applied the RDTM to various nonlinear evolution equations and compared the obtained results with the exact solution. The main advantage of the RDTM is the fact that it provides its user with an analytical approximation, in many cases an exact solution. The solution procedure of the RDTM is simpler than traditional DTM, and the amount of computation required in RDTM is much less than traditional DTM.

\section{Reduced Differential Transform Method (RDTM)}

Consider a function of two variables $u(x, t)$ and suppose that it can be represented as a product of two single-variable functions, i.e., $u(x, t)=f(x) g(t)$. Based on the properties of one-dimensional differential transform, the function $u(x, t)$ can be represented as follows:

$$
u(x, t)=\left(\sum_{i=0}^{\infty} F(i) x^{i}\right)\left(\sum_{j=0}^{\infty} G(j) t^{j}\right)=\sum_{k=0}^{\infty} U_{k}(x) \cdot t^{k}
$$

where $U_{k}(x)$ is called $t$-dimensional spectrum function $u(x, t)$.

The basic definitions of reduced differential transform method [32-34] are introduced as follows:

Definition 2.1. If a function $u(x, t)$ is analytic and differentiated continuously with respect to time $t$ and space $x$ in the domain of interest then let

$$
U_{k}(x)=\frac{1}{k !}\left[\frac{\partial^{k}}{\partial t^{k}} u(x, t)\right]_{t=0}, k \geq 0, k \in N
$$

where the $t$-dimensional spectrum function $U_{k}(x)$ is the transformed function and $u(x, t)$ is the original function. 
Definition 2.2. The differential inverse transform of $U_{k}(x)$ is defined as follows:

$$
u(x, t)=\sum_{k=0}^{\infty} U_{k}(x) \cdot t^{k}
$$

From Eq.(2) and Eq.(3), we get

$$
u(x, t)=\sum_{k=0}^{\infty} \frac{t^{k}}{k !}\left[\frac{\partial^{k}}{\partial t^{k}} u(x, t)\right]_{t=0}
$$

The following theorems that can be deduced from Eqs.(2-4) are given below:

Theorem 2.1. If $f(x, t)=a g(x, t) \pm b h(x, t)$, then

$$
F_{k}(x)=a G_{k}(x) \pm b H_{k}(x) \text {, where } a \text { and } b \text { are constant } .
$$

Theorem 2.2. If $f(x, t)=x^{m} t^{n}$, then $F_{k}(x)=x^{m} \delta(k-n)$ where $\delta(j)=\left\{\begin{array}{l}1, j=0 \\ 0, j \neq 0\end{array}\right.$.

Theorem 2.3. If $f(x, t)=x^{m} t^{n} g(x, t)$, then $F_{k}(x)=x^{m} G_{k-n}(x)$

Theorem 2.4. If $f(x, t)=g(x, t) \cdot h(x, t)$, then $F_{k}(x)=\sum_{l=0}^{k} G_{l}(x) \cdot H_{k-l}(x)$

Theorem 2.5. If $f(x, t)=g_{1}(x, t) \cdot g_{2}(x, t) \mathrm{L} g_{n-1}(x, t) \cdot g_{n}(x, t)$, then

$$
F_{k}(x)=\sum_{k_{n-1}}^{k} \sum_{k_{n-2}}^{k_{n-2}} \mathrm{~L} \sum_{k_{2}}^{k_{3}} \sum_{k_{1}}^{k_{2}} G_{1, k_{1}}(x) \cdot G_{2, k_{2}-k_{1}}(x) \mathrm{L} G_{n-1, k_{n-1}-k_{n-2}}(x) \cdot G_{n, k-k_{n-1}}(x)
$$

Theorem 2.6. If $f(x, t)=\frac{\partial^{n}}{\partial t^{n}} g(x, t)$, then $F_{k}(x)=\frac{(k+n) !}{k !} G_{k+n}(x)$

Theorem 2.7. If $f(x, t)=\frac{\partial^{n}}{\partial x^{n}} g(x, t)$, then $F_{k}(x)=\frac{\partial^{n}}{\partial x^{n}} G_{k}(x)$

Theorem 2.8. If $f(x, t)=\frac{\partial^{n+m}}{\partial x^{n} \partial t^{m}} g(x, t)$, then $F_{k}(x)=\frac{\partial^{n}}{\partial x^{n}}\left(\frac{(k+m) !}{k !} G_{k+m}(x)\right)$

\section{Solution of The Nonlinear Evolution Equations By The RDTM}

In this section, the RDTM is used to find approximate solutions of some nonlinear evolution equations, namely, Korteweg-de Vries Burgers' (KdVB) equations, Drinefel'd-Sokolov-Wilson equations, coupled Burgers equations and modified Boussinesq equation. 


\subsection{RDTM for Korteweg-de Vries (KdV) and Korteweg-de Vries-Burgers (KdVB) Equations}

Let us first consider the KdVB equation has the form

$$
u_{t}+\varepsilon u u_{x}-v u_{x x}+\mu u_{x x x}=0,
$$

where $\varepsilon, v$ and $\mu$ are constants. We will investigate the two cases, the first one is the $\mathrm{KdV}$ equation (in case of $v=0$ ) and the second one is the KdVB (in case of $\varepsilon=1)$.

Case 1. We consider the $\mathrm{KdV}$ equation in Eq.(5) for $\varepsilon=6, v=0$ and $\mu=1$

$$
u_{t}+6 u u_{x}+u_{x x x}=0,
$$

subject to the initial condition ;

$$
u(x, 0)=\frac{1}{2} \sec h^{2}\left(\frac{x}{2}\right)
$$

The exact solution of this problem is

$$
u(x, t)=\frac{1}{2} \sec h^{2}\left(\frac{1}{2}(x-t)\right)
$$

Applying the above theorems we obtain following recurrence relation for the $\mathrm{KdV}$ equation.

$$
(k+1) U_{k+1}(x)=-6 \cdot \sum_{l=0}^{k} U_{l}(x) \frac{\partial}{\partial x} U_{k-l}(x)-\frac{\partial^{3}}{\partial x^{3}} U_{k}(x)
$$

Using Eq.(2), the initial condition given in Eq. (7) can be transformed as,

$$
U_{0}(x)=\frac{1}{2} \sec h^{2}\left(\frac{x}{2}\right)
$$

Substituting Eq.(10) into Eq.(9) and by straightforward iterative steps, we get the following $U_{k}(x)$ (for $\mathrm{k}=0,1,2, \ldots, \mathrm{n}$ ) values.

$$
\begin{aligned}
& U_{0}(x)=\frac{1}{2} \sec ^{2}(x / 2), \\
& U_{1}(x)=\frac{1}{2} \tanh (x / 2) \sec h(x / 2), \\
& U_{2}(x)=\frac{1}{8}\left\{2 \sec h^{2}(x / 2)-3 \sec h^{4}(x / 2)\right\} \\
& U_{3}(x)=\frac{1}{12}\left\{\tanh (x / 2)\left[\operatorname{sech} h^{2}(x / 2)-3 \sec h^{4}(x / 2)\right]\right\}
\end{aligned}
$$


and so on, in the same manner, the rest of components can be obtained using MAPLE.

Using the inverse transformation Eq.(3), we get the approximate solution as,

$$
\begin{aligned}
\tilde{u}(x, t) & =\frac{1}{2} \sec h^{2}(x / 2)+\left(\frac{1}{2} \tanh (x / 2) \sec h(x / 2)\right) t \\
& +\left(\frac{1}{8}\left\{2 \sec h^{2}(x / 2)-3 \sec h^{4}(x / 2)\right\}\right) \mathrm{t}^{2} \\
& +\left(\frac{1}{12}\left\{\tanh (x / 2)\left[\sec h^{2}(x / 2)-3 \sec h^{4}(x / 2)\right]\right\}\right) \mathrm{t}^{3}+\cdots
\end{aligned}
$$

The behavior of the approximate solution obtained by RDTM with the exact solution (Eq.(8)) for different values of times is shown in Fig.1. The comparison shows that the two solutions obtained are in excellent agreement.

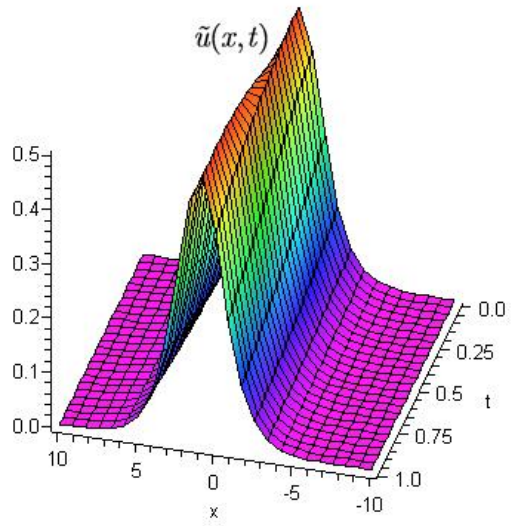

(a)

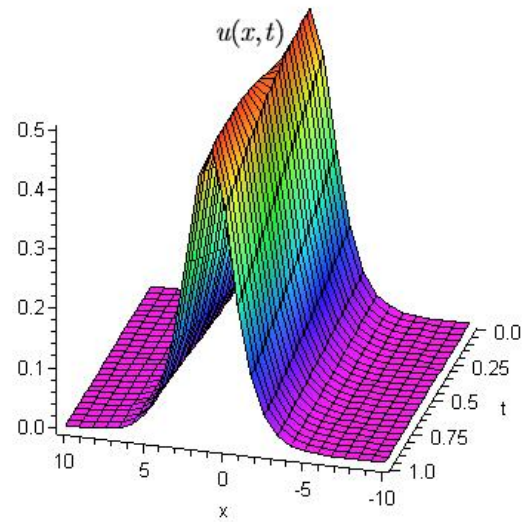

(b)

Fig. 1.(a) The approximate solution $\tilde{u}(x, t)$ obtained by RDTM with different values of time.(b) The exact solution $u(x, t)$ with different values of time.

Case 2. Now, we consider the KdVB equation in Eq.(5) for $\varepsilon=1$

$$
u_{t}+u u_{x}-v u_{x x}+\mu u_{x x x}=0,
$$

subject to the initial condition ;

$$
u(x, 0)=-\frac{6 v^{2}}{25 \mu}\left[1+\tan h \gamma-\frac{1}{2} \sec h^{2} \gamma\right]
$$

where $\gamma=\frac{v x}{10 \mu}$.

The exact solution of this problem is 


$$
u(x, t)=-\frac{6 v^{2}}{25 \mu}\left[1+\tan h(\xi)-\frac{1}{2} \sec h^{2}(\xi)\right]
$$

where $\xi=\frac{v}{10 \mu}\left(x+\frac{6 v^{2}}{25 \mu} t\right)$.

According to the above theorems, we have the following recurrence relation for the KdVB equation:

$$
(k+1) U_{k+1}(x)=v \frac{\partial^{2}}{\partial x^{2}} U_{k}(x)-\sum_{l=0}^{k} U_{l}(x) \frac{\partial}{\partial x} U_{k-l}(x)-\mu \frac{\partial^{3}}{\partial x^{3}} U_{k}(x)
$$

From Eq.(2), the initial condition given in Eq. (14) can be transformed at $t=0$ as

$$
U_{0}(x)=-\frac{6 v^{2}}{25 \mu}\left[1+\tan h \gamma-\frac{1}{2} \sec h^{2} \gamma\right]
$$

Substituting Eqs.(17) into Eq.(16), we get the following $U_{k}(x)$ (for $\left.\mathrm{k}=0,1,2, \ldots, \mathrm{n}\right)$ values.

$$
\begin{aligned}
& U_{0}(x)=-\frac{6 v^{2}}{25 \mu}\left(1+\tanh \gamma-\frac{1}{2} \sec h^{2} \gamma\right), \\
& U_{1}(x)=-\frac{18 v^{5}}{3125 \mu^{3}}\left((1+\tanh \gamma) \cdot \sec h^{2} \gamma\right) \\
& U_{2}(x)=\frac{27 v^{8}}{390625 \mu^{5}}\left(\left(2+2 \tanh \gamma-3 \sec h^{2} \gamma\right) \cdot \sec h^{2} \gamma\right), \\
& U_{3}(x)=-\frac{54 v^{11}}{48828125 \mu^{7}}\left(\left(2+2 \tanh \gamma-6 \tanh \gamma \sec h^{2} \gamma-3 \sec h^{2} \gamma\right) \cdot \sec h^{2} \gamma\right)
\end{aligned}
$$

where $\gamma=\frac{v x}{10 \mu}$.

Using the inverse transformation Eq.(3), we get the approximate solution as,

$$
\begin{aligned}
\tilde{u}(x, t) & =-\frac{6 v^{2}}{25 \mu}\left(1+\tanh \gamma-\frac{1}{2} \sec h^{2} \gamma\right)-\frac{18 v^{5}}{3125 \mu^{3}}\left((1+\tanh \gamma) \cdot \sec h^{2} \gamma\right) t \\
& +\frac{27 v^{8}}{390625 \mu^{5}}\left(\left(2+2 \tanh \gamma-3 \sec h^{2} \gamma\right) \cdot \sec h^{2} \gamma\right) \mathrm{t}^{2} \\
& -\frac{54 v^{11}}{48828125 \mu^{7}}\left(\left(2+2 \tanh \gamma-6 \tanh \gamma \sec h^{2} \gamma-3 \sec h^{2} \gamma\right) \cdot \sec h^{2} \gamma\right) \mathrm{t}^{3}+\cdots
\end{aligned}
$$

The behavior of the approximate solution with the exact solution (Eq.(15)) for different values of times is shown in Fig.2. 


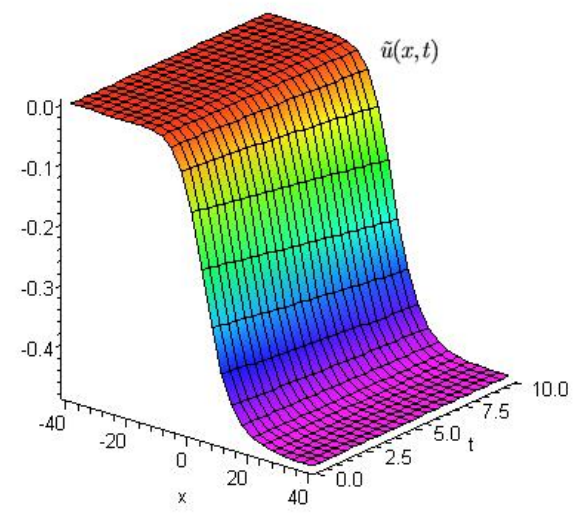

(a)

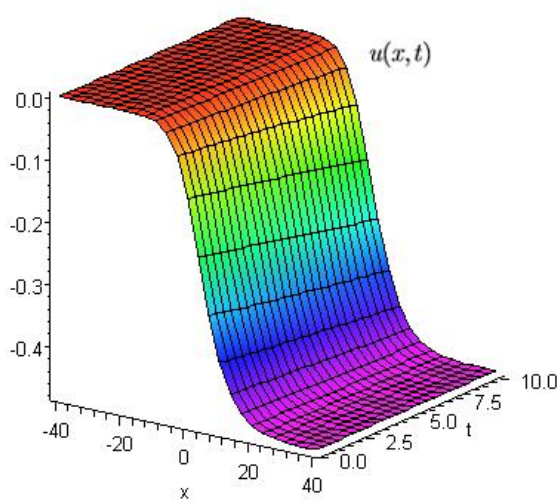

(b)

Fig. 2. (a) The approximate solution $\tilde{u}(x, t)$ obtained by RDTM with different values of time with fixed values $v=1, \mu=1$. (b) The exact solution $u(x, t)$ with different values of time with fixed values $v=1, \mu=1$.

\subsection{RDTM for Drinefel'd-Sokolov-Wilson Equations}

In this section, we consider the Drinefel'd-Sokolov-Wilson equations

$$
\begin{aligned}
& u_{t}+p v v_{x}=0 \\
& v_{t}+q v_{x x x}+r u v_{x}+s v u_{x}=0
\end{aligned}
$$

where $\mathrm{p}, \mathrm{q}, \mathrm{r}$, and $\mathrm{s}$ are arbitrary constants. For $p=q=r=1$ the initial conditions of $u(x, t)$ and $v(x, t)$ are given by

$$
u(x, 0)=2 \operatorname{sech}^{2} x, \quad v(x, 0)=2 \sec h x
$$

and the exact solutions are

$$
u(x, t)=2 \sec h^{2}(x-t), \quad v(x, t)=2 \sec h(x-t)
$$

Using above theorems we get following recurrence relations;

$$
\begin{gathered}
(k+1) U_{k+1}(x)=-p \sum_{l=0}^{k} V_{l}(x) \frac{\partial}{\partial x} V_{k-l}(x) \\
(k+1) V_{k+1}(x)=-q \frac{\partial^{3}}{\partial x^{3}} U_{k}(x)-r \sum_{l=0}^{k} U_{l}(x) \frac{\partial}{\partial x} V_{k-l}(x)-s \sum_{l=0}^{k} V_{l}(x) \frac{\partial}{\partial x} U_{k-l}(x)
\end{gathered}
$$

From Eq.(2), the initial condition given in Eq. (22) can be transformed as

$$
U_{0}(x)=2 \sec h^{2} x, V_{0}(x)=2 \sec h x
$$


Substituting Eqs.(26) into Eqs.(24-25), we get the following $U_{k}(x)$ and $V_{k}(x)$ (for $\mathrm{k}=0,1,2, \ldots, \mathrm{n}$ ) values.

$$
\begin{array}{ll}
U_{0}(x)=2 \sec h^{2} x, & V_{0}(x)=2 \sec h x, \\
U_{1}(x)=4 \sec h^{2} x \cdot \tan h x, & V_{1}(x)=2 \sec h x \cdot \tan h x \\
U_{2}(x)=4 \sec h^{2} x-6 \sec h^{4} x, & V_{2}(x)=\sec h^{2} x-2 \sec h^{3} x \\
U_{3}(x)=\frac{8}{3} \sec h^{4} x \cdot \tan h x \cdot\left(\cosh ^{2} x-3\right), & V_{3}(x)=\frac{1}{3} \sec h^{3} x \cdot \tan h x \cdot\left(\cosh ^{2} x-6\right)
\end{array}
$$

Then, using the inverse transformation Eq.(3), we obtain approximate solution as,

$$
\begin{aligned}
\tilde{u}(x, t) & =2 \sec h^{2} x+\left(4 \sec h^{2} x \cdot \tan h x\right) t+\left(4 \sec h^{2} x-6 \sec h^{4} x\right) t^{2} \\
& +\left(\frac{8}{3} \sec h^{4} x \cdot \tan h x \cdot\left(\cosh ^{2} x-3\right)\right) t^{3}+\cdots \\
\tilde{v}(x, t) & =2 \sec h x+(2 \sec h x \cdot \tan h x) t+\left(\sec h^{2} x-2 \sec h^{3} x\right) t^{2} \\
& +\left(\frac{1}{3} \sec h^{3} x \cdot \tan h x \cdot\left(\cosh ^{2} x-6\right)\right) t^{3}+\cdots
\end{aligned}
$$

The graphical behavior of the approximate solutions obtained by RDTM with the exact solutions (Eqs.(26)) for different values of times is shown in Fig.3.
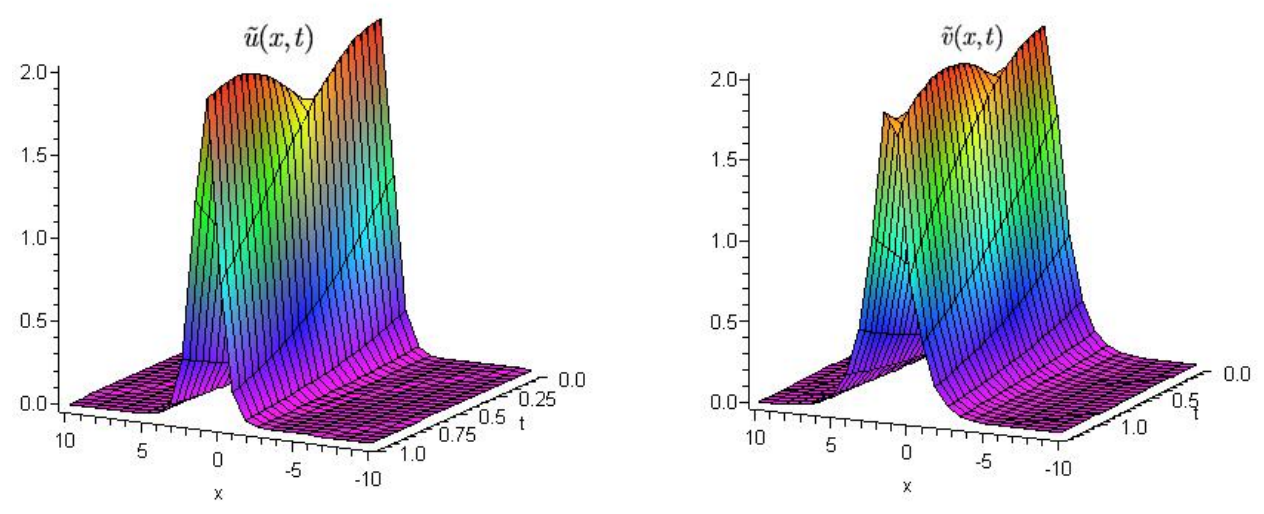


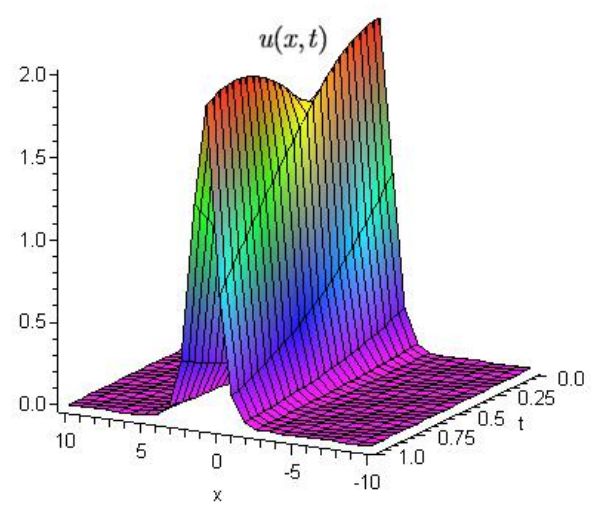

(a)

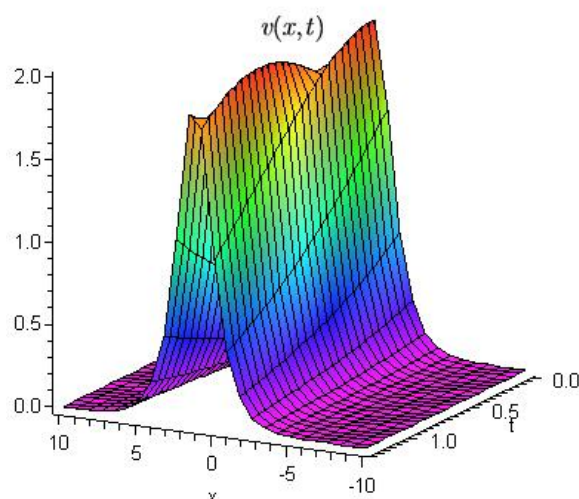

(b)

Fig. 3. (a) The approximate solutions $\tilde{u}(x, t)$ and $\tilde{v}(x, t)$ obtained by RDTM with different values of time. (b) The exact solutions $u(x, t)$ and $v(x, t)$ with different values of time. (For $p=q=r=1$ )

\subsection{RDTM for coupled Burgers Equations}

Now, we will consider the system of Burgers' equations in the operator form

$$
\begin{aligned}
& u_{t}-u_{x x}-2 u u_{x}+(u v)_{x}=0, \\
& v_{t}-v_{x x}-2 v v_{x}+(u v)_{x}=0,
\end{aligned}
$$

subject to the initial conditions

$$
u(x, 0)=\sin (x), \quad v(x, 0)=\sin (x),
$$

The exact solutions of this system are

$$
u(x, t)=e^{-t} \sin (x), \quad v(x, t)=e^{-t} \sin (x),
$$

According to the above theorems, we have the following recurrence relation for the system of Burgers' equations:

$$
\begin{aligned}
& (k+1) U_{k+1}(x)=\frac{\partial^{2}}{\partial x^{2}} U_{k}(x)+2 \sum_{l=0}^{k} U_{l}(x) \frac{\partial}{\partial x} U_{k-l}(x)-\frac{\partial}{\partial x}\left(\sum_{l=0}^{k} U_{l}(x) V_{k-l}(x)\right) \\
& (k+1) V_{k+1}(x)=\frac{\partial^{2}}{\partial x^{2}} V_{k}(x)+2 \sum_{l=0}^{k} V_{l}(x) \frac{\partial}{\partial x} V_{k-l}(x)-\frac{\partial}{\partial x}\left(\sum_{l=0}^{k} U_{l}(x) V_{k-l}(x)\right)
\end{aligned}
$$

From Eq.(2), the initial condition given in Eq. (32) can be transformed as;

$$
U_{0}(x)=\sin x, \quad V_{0}(x)=\sin x
$$


Substituting Eqs.(36) into Eqs.(34-35) and by straightforward iterative steps, we get the following $U_{k}(x)$ and $V_{k}(x)$ (for $\mathrm{k}=0,1,2, \ldots, \mathrm{n}$ ) values.

$$
\begin{aligned}
& U_{0}(x)=V_{0}(x)=\sin x, U_{1}(x)=V_{1}(x)=-\sin x \\
& U_{2}(x)=V_{2}(x)=\frac{1}{2} \sin x, U_{3}(x)=V_{3}(x)=-\frac{1}{6} \sin x \\
& U_{4}(x)=V_{4}(x)=\frac{1}{24} \sin x, \ldots
\end{aligned}
$$

Then, using the inverse transformation Eq.(3), we get approximate solution as,

$$
\begin{aligned}
\tilde{u}(x, t) & =\sin x-t \sin x+\frac{1}{2} \mathrm{t}^{2} \sin x-\frac{1}{6} \mathrm{t}^{3} \sin x \\
& +\frac{1}{24} \mathrm{t}^{4} \sin x-\frac{1}{120} \mathrm{t}^{5} \sin x+\cdots \\
& =\left(1-t+\frac{1}{2} \mathrm{t}^{2}-\frac{1}{6} \mathrm{t}^{3}+\frac{1}{24} \mathrm{t}^{4}-\frac{1}{120} \mathrm{t}^{5}+\cdots\right) \sin x \\
\tilde{v}(x, t) & =\sin x-t \sin x+\frac{1}{2} \mathrm{t}^{2} \sin x-\frac{1}{6} \mathrm{t}^{3} \sin x \\
& +\frac{1}{24} \mathrm{t}^{4} \sin x-\frac{1}{120} \mathrm{t}^{5} \sin x+\cdots \\
& =\left(1-t+\frac{1}{2} \mathrm{t}^{2}-\frac{1}{6} \mathrm{t}^{3}+\frac{1}{24} \mathrm{t}^{4}-\frac{1}{120} \mathrm{t}^{5}+\cdots\right) \sin x
\end{aligned}
$$

which are Taylor series of Eqs.(34).

\subsection{RDTM for Modified Boussinesq Equation}

Finally, we consider the following general equation

$$
u_{t t}+\alpha u_{x x t}+\beta u_{x x x x}+\gamma\left(u^{n}\right)_{x x}=0
$$

where $\alpha, \beta, \gamma$ and $n$ are constants.

This equation is called the high-order modified Boussinesq equation with the damping term $u_{x x t}$. It appears in several domains of mathematics and physics.

Now, we will consider the cubic modified Boussinesq equation $(\alpha=1, \beta=2 / 9, \gamma=-1$ and $n=3$ )

$$
u_{t t}+u_{x x t}+\frac{2}{9} u_{x x x x}-\left(u^{3}\right)_{x x}=0
$$

with initial conditions 


$$
u(x, 0)=1+\tanh \left(\frac{3}{2} x\right), u_{t}(x, 0)=-3 \sec h^{2}\left(\frac{3}{2} x\right)
$$

The exact solution of this problem is

$$
u(x, t)=1+\tanh \left(\frac{3}{2} x-3 t\right)
$$

Using above theorems we obtain following recurrence relation;

$$
\begin{aligned}
(k+1)(k+2) U_{k+2}(x) & =-(k+1) \frac{\partial^{2}}{\partial x^{2}} U_{k+1}(x)-\frac{2}{9} \frac{\partial^{4}}{\partial x^{4}} U_{k}(x) \\
& +\frac{\partial^{2}}{\partial x^{2}}\left(\sum_{l=0}^{k} \sum_{r=0}^{l} U_{r}(x) U_{l-r}(x) U_{k-l}(x)\right)
\end{aligned}
$$

From Eq.(2), the initial conditions given in Eq. (42) can be transformed as

$$
U_{0}(x)=1+\tanh \left(\frac{3}{2} x\right), U_{1}(x)=-3 \sec h^{2}\left(\frac{3}{2} x\right)
$$

Substituting Eqs.(45) into Eq.(44) and by straightforward iterative steps, we get the following $U_{k}(x)$ (for $\left.\mathrm{k}=0,1,2, \ldots, \mathrm{n}\right)$ values.

$$
\begin{aligned}
& U_{0}(x)=1+\tanh \left(\frac{3}{2} x\right), U_{1}(x)=-3 \operatorname{sech} h^{2}\left(\frac{3}{2} x\right) \\
& U_{2}(x)=-9 \tanh \left(\frac{3}{2} x\right) \operatorname{sech}^{2}\left(\frac{3}{2} x\right) \\
& U_{3}(x)=\operatorname{sech} 2\left(\frac{3}{2} x\right)\left(-18+27 \operatorname{sech} h^{2}\left(\frac{3}{2} x\right)\right) \\
& U_{4}(x)=\tanh \left(\frac{3}{2} x\right) \operatorname{sech} h^{2}\left(\frac{3}{2} x\right)\left(-27+81 \operatorname{sech}^{2}\left(\frac{3}{2} x\right)\right), \ldots
\end{aligned}
$$

Then, using the inverse transformation Eq.(3), we get the approximate solution as,

$$
\begin{aligned}
\tilde{u}(x, t) & =1+\tanh \left(\frac{3}{2} x\right)-3 \sec h^{2}\left(\frac{3}{2} x\right) \cdot t-9 \tanh \left(\frac{3}{2} x\right) \operatorname{sech}^{2}\left(\frac{3}{2} x\right) \cdot t^{2} \\
& +\operatorname{sech} h^{2}\left(\frac{3}{2} x\right)\left(-18+27 \sec h^{2}\left(\frac{3}{2} x\right)\right) \cdot t^{3} \\
& +\tanh \left(\frac{3}{2} x\right) \operatorname{sech} h^{2}\left(\frac{3}{2} x\right)\left(-27+81 \sec h^{2}\left(\frac{3}{2} x\right)\right) \cdot t^{4}+\cdots
\end{aligned}
$$


The behavior of the approximate solution with the exact solutions (Eq.(43)) for different values of times is shown in Fig.4.

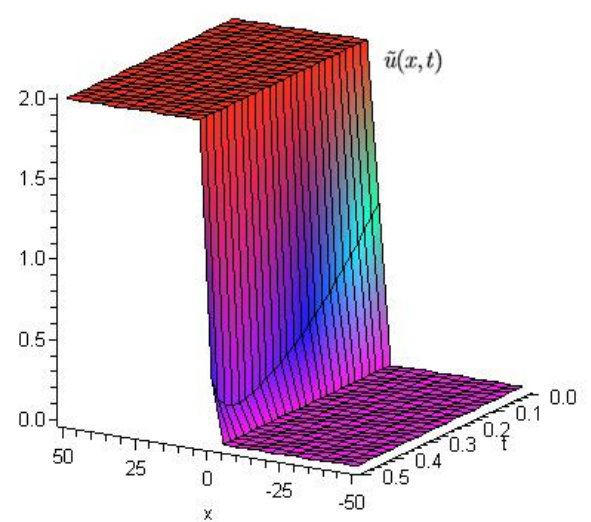

(a)

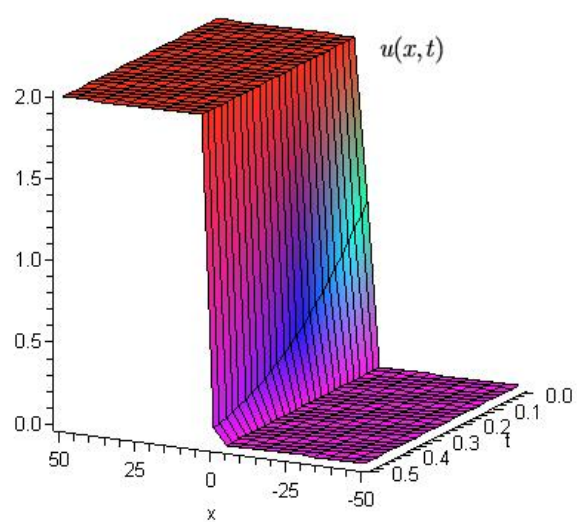

(b)

Fig. 4. (a) The approximate solution $\tilde{u}(x, t)$ obtained by RDTM with different values of time. (b) The exact solution $u(x, t)$ with different values of time.

\section{Conclusion}

In this paper, the reduced transform method (RDTM) has been successfully applied to nonlinear evolution equations. The approximate solutions of Kortewegde Vries Burgers' (KdVB) equation, Drinefel'd-Sokolov-Wilson equations, coupled Burgers equations and modified Boussinesq equation are obtained. The approximate solutions obtained are in good agreement with the known exact solutions. The results show that the RDTM is an efficient approach for the solution of such type of nonlinear equations. The main advantage of the RDTM is to provide the user an analytical approximation to the solution, in many cases, an exact solution, in a rapidly convergent sequence with elegantly computed terms. The solution procedure of the RDTM is simple than other existing techniques.

\section{References}

[1] J.H. He, "Variational iteration method-a kind of non-linear analytical technique: some examples", Int. J. Noniinear Mech., Vol.34, No.4 (1999), pp.699-708.

[2] A.A. Soliman and M.A. Abdou, "Numerical solutions of nonlinear evolution equations using variational iteration method", J. Comput. and Appl. Math., Vol.207, No.1 (2007), pp.111-120. 
[3] D.D. Ganji, H. Tari and M.B. Jooybari, "Variational iteration method and homotopy perturbation method for nonlinear evolution equations", Comput. Math. Appl., Vol.54, No.7-8 (2007), pp.1018-1027.

[4] J.H. He, "Application of homotopy perturbation method to nonlinear wave equations", Chaos, Solitons and Fractals, Vol.26, No.3 (2005), pp.695-700.

[5] J.H. He, "New interpretation of homotopy perturbation method", Int. J. Modern Phys. B, Vol.20, No.18 (2006), pp.2561-2568.

[6] L. Song and H. Zhang, "Application of the extended homotopy perturbation method to a kind of nonlinear evolution equations", Appl. Math. Comput., Vol.197, No.1 (2008), pp.87-95.

[7] W. Malfliet and W. Hereman, "The tanh method: I. exact solutions of nonlinear evolution and wave equations", Phys. Scr., Vol.54 (1996), pp.563568.

[8] E.G. Fan, "Extended tanh-function method and its applications to nonlinear equations", Phys. Lett. A, Vol.277 (2000), pp.212-218.

[9] Z.Y. Yan, "New explicit travelling wave solutions for two new integrable coupled nonlinear evolution equations", Phys. Lett. A, Vol.292 (2001), pp.100-106.

[10] A.Bekir, "Applications of the extended tanh method for coupled nonlinear evolution equations", Commun. Nonlinear Sci. Numer. Simul., Vol.13, No.9 (2008), pp.1748-1757.

[11] S.A. El-Wakil and M.A. Abdou, "Modified extended tanh-function method for solving nonlinear partial differential equations", Chaos, Solitons Fractals, Vol.31, No.5 (2007), pp.1256-1264.

[12] J.L. Zhang, M.L. Wang, Y.M. Wang and Z.D. Fang, "The improved Fexpansion method and its applications", Phys. Lett. A, Vol.350 (2006), pp.103-109.

[13] M.A. Abdou, "The extended F-expansion method and its application for a class of nonlinear evolution equations", Chaos, Solitons Fractals, Vol.31 (2007), pp. 95-104.

[14] A.M. Wazwaz, "A sine-cosine method for handling nonlinear wave equations", Math. Comput. Modelling, Vol.40 (2004), pp.499-508.

[15] A.M. Wazwaz, "Solitary wave solutions and periodic solutions for higherorder nonlinear evolution equations", Appl. Math. Comput., Vol.181, No.2 (2006), pp.1683-1692.

[16] E. Yusufoglu and A. Bekir, "Solitons and periodic solutions of coupled nonlinear evolution equations by using the sine-cosine method", Int. J. Comput. Math., Vol.83, No.12 (2006), pp.915-924.

[17] F. Taşcan and A.Bekir, "Analytic solutions of the (2+1)-dimensional nonlinear evolution equations using the sine-cosine method", Appl. Math. Comput., Vol.215, No.8 (2009), pp.3134-3139.

[18] A.M. Wazwaz, "The Hirota's direct method for multiple-soliton solutions for three model equations of shallow water waves", Appl. Math. Comput., Vol.201, No.1-2 (2008), pp.489-503. 
[19] A.M. Wazwaz, "Multiple-soliton solutions for the KP equation by Hirota's bilinear method and by the tanh-coth method", Appl. Math. Comput., Vol.190, No.1 (2007), pp.633-640.

[20] H. Zhang, "New exact Jacobi elliptic function solutions for some nonlinear evolution equations", Chaos, Solitons and Fractals, Vol.32, No.2 (2007), pp.653-660.

[21] S.A. El-Wakil, M.A. Madkour and M.A. Abdou, "New traveling wave solutions for nonlinear evolution equations", Phys. Lett. A, Vol.365, No.5-6 (2007), pp.429-438.

[22] Y.Yu, Q.Wang and H. Zhang, "The extended Jacobi elliptic function method to solve a generalized Hirota-Satsuma coupled KdV equations", Chaos, Solitons Fractals, Vol.26, No.5 (2005), pp.1415-1421.

[23] E. Fan and H. Zhang, "A note on the homogeneous balance method", Phys. Lett. A, Vol.246 (1998), pp.403-406.

[24] M.L. Wang, "Exact solutions for a compound KdV-Burgers equation", Phys. Lett. A, Vol.213 (1996), pp.279-287.

[25] M. Wang, X. Li and J. Zhang, "The $\left(\mathrm{G}^{\prime} / \mathrm{G}\right)$-expansion method and travelling wave solutions of nonlinear evolution equations in mathematical physics", Phys. Lett. A, Vol.372 (2008), pp.417-423.

[26] A. Bekir, "Application of the $\left(\mathrm{G}^{\prime} / \mathrm{G}\right)$-expansion method for nonlinear evolution equations", Phys. Lett. A, Vol.372, No.19 (2008), pp.3400-3406.

[27] S. Kutluay, A. Esen and O. Tasbozan, "The $\left(\mathrm{G}^{\prime} / \mathrm{G}\right)$-expansion method for some nonlinear evolution equations", Appl. Math. Comput., Vol.217, No.1 (2010), pp.384-391.

[28] J.H. He and X.H. Wu, "Exp-function method for nonlinear wave equations", Chaos, Solitons Fractals, Vol.30 (2006), pp.700-708.

[29] A. Ebaid, "Exact solitary wave solutions for some nonlinear evolution equations via Exp-function method", Phys. Lett. A, Vol.365, No.3 (2007), pp.213-219.

[30] A. Bekir and A. Boz, "Exact solutions for nonlinear evolution equations using Exp-function method", Phys. Lett. A, Vol.372 (2008), pp.1619-1625.

[31] A. Ebaid, "Exact solitary wave solutions for some nonlinear evolution equations via Exp-function method", Phys. Lett. A, Vol.365 (2007), pp.213219.

[32] Y.Keskin and G.Oturanç, "Reduced differential transform method for partial differential equations", Int J. Nonlinear Sci Numer. Simul, Vol.10, No.6 (2009), pp.741-749.

[33] Y.Keskin and G.Oturanç, "The reduced differential transform method: a new approach to factional partial differential equations", Nonlinear Sci. Lett. A, Vol.1, No.2 (2010), pp. 207-217.

[34] K. Yıldırım, B. İbiş and M. Bayram, "New solutions of the non linear Fisher type equations by the reduced differential transform", Nonlinear Sci. Lett. A, Vol.3, No.1 (2012), pp.29-36. 
[35] A. Reza and G. Masoud, "Extended two-dimensional DTM and its a pplication on nonlinear PDEs with proportional delay", Int. J. Comput. Math., Vol.88, No.8 (2011), pp.1749-1762.

[36] D. Kaya, "An application of the decomposition method for the $\mathrm{KdVb}$ equation", Appl. Math . Comput., Vol.152 (2004), pp.279-288.

[37] R. Yao and Z. Li, "New exact solutions for three nonlinear evolution equations", Phys. Lett. A, Vol.297 (2002), pp.196-204.

[38] M. Dehghan, A. Hamidi and M. Shakourifar, "The solution of coupled Burgers' equations using Adomian-Pade technique", Appl. Math. Comput., Vol.189, No.2 (2007), pp.1034-1047. 